\title{
Estimation of Genetic Parameters for Feeding Pattern Traits and Its Relationship to Feed Efficiency and Production Traits in Duroc Pigs
}

\author{
Kier G. Santiago ${ }^{1,2}$, Sung-Hoon Kim ${ }^{3}$, Bryan Irvine Lopez ${ }^{4} \oplus$, Dong-Hui Lee ${ }^{1}$, Young-Gyu Cho ${ }^{1}$, Yu-Na Song ${ }^{1}$ \\ and Kang-Seok Seo ${ }^{1, *}$ \\ 1 Department of Animal Science and Technology, Sunchon National University, Suncheon 57922, Korea; \\ santiagokier2015@gmail.com (K.G.S.); a3832737@naver.com (D.-H.L.); dudrb1992@naver.com (Y.-G.C.); \\ syn1010@naver.com (Y.-N.S.) \\ 2 Department of Animal Science, Central Luzon State University, Science City of Muñoz 3120, Philippines \\ 3 Pig Gene Korea Co., Ltd., Yongin 16866, Korea; drskim@naver.com \\ 4 Division of Animal Genomics and Bioinformatics, National Institute of Animal Science, Rural Development \\ Administration, Wanju 55365, Korea; irvinelopez@korea.kr \\ * Correspondence: sks@sunchon.ac.kr
}

\section{check for}

updates

Citation: Santiago, K.G.; Kim, S.-H.; Lopez, B.I.; Lee, D.-H.; Cho, Y.-G.;

Song, Y.-N.; Seo, K.-S. Estimation of Genetic Parameters for Feeding Pattern Traits and Its Relationship to Feed Efficiency and Production Traits in Duroc Pigs. Agriculture 2021, 11, 850. https://doi.org/10.3390/ agriculture11090850

Academic Editor:

Javier Álvarez-Rodríguez

Received: 30 July 2021

Accepted: 1 September 2021

Published: 6 September 2021

Publisher's Note: MDPI stays neutral with regard to jurisdictional claims in published maps and institutional affiliations.

Copyright: (c) 2021 by the authors. Licensee MDPI, Basel, Switzerland. This article is an open access article distributed under the terms and conditions of the Creative Commons Attribution (CC BY) license (https:/ / creativecommons.org/licenses/by/ $4.0 /)$.

\begin{abstract}
This study was conducted to estimate the genetic parameters of different feeding pattern traits, including average daily feed intake (ADFI), average occupation time per day (AOTD), average occupation time per visit (AOTV), average daily feeding rate (ADFR), average feeding rate per feeding visit (AFRV), average feed intake per feeding visit (AFIV), and average number of visits per day (ANVD), and their genetic relationship to production traits, such as on-test average daily gain (ADG), backfat thickness (BFT), loin muscle area (LMA), lean percentage (LP), and feed efficiency traits, such as feed conversion ratio (FCR) and five measures of residual feed intake (RFI1 to RFI5), in Duroc pigs (DD). The non-heritable common spatial pen effect was also estimated in all studied traits. The feeding pattern traits used in this study were derived from filtered feeding visits of 602 DD pigs. Using three animal models and the REML method, the genetic parameters revealed low to moderate heritability for ADFI (0.19 to 0.32$)$ and AFIV (0.18 to 0.33), moderate heritability for ANVD (0.28 to 0.35) and AOTV (0.21 to 0.31), and high heritability for AOTD (0.73), ADFR (0.62 to 0.64), and AFRV (0.59 to 0.63). The addition of a common spatial pen effect in models 2 and 3 had a substantial impact, ranging from $8 \%$ to $23 \%$, on the total variability of most feeding pattern traits, with the exception of AOTD, which only had a percentage variance of $0.30 \%$ due to the pen effect. The genetic and phenotypic correlation revealed that ADFI had consistent moderate to high genetic and phenotypic correlation with production and feed efficiency (FE) traits. However, selection against ADFI would negatively affect on-test ADG. Interestingly, the AOTD had no genetic correlation with ADG (0.04), low to moderate positive genetic correlation with FCR (0.27) and all RFI measures (0.24 to 0.33$)$, and moderate negative correlation with LP (-0.39), indicating that selection for DD pigs with lower AOTD would not influence on-test ADG but may increase LP and improve feed efficiency by lowering FCR and all RFI measures. However, the corresponding phenotypic correlation of AOTD with production and feed efficiency traits was mostly weak, which can be attributed to the low residual or environmental correlation between these correlated traits. At the genetic level, the feeding pattern traits showed potential in improving feed efficiency and production traits. However, further studies are needed to evaluate their impact at phenotypic level.
\end{abstract}

Keywords: feeding pattern; heritability; genetic correlation; Duroc pigs

\section{Introduction}

In early feeding behavior studies, the feed intake of pigs was only able to be measured by housing the animals individually, with the result that the pigs ate more, grew faster, 
and became fatter than pigs housed in groups [1]. However, pigs in actual commercial operation are housed in groups which, prior to the development of automatic electronic feeders, made the recording of feed intake challenging. According to McSweeny (2001) [2], the three most commonly used electronic feeders developed for pigs are the ACEMA 48 feeders developed in France, the IVOG feeders (individual voluntary feed intake recording in group pens) developed in Wageningen, The Netherlands, and the FIRE (feed intake recording equipment) feeders originally developed in England. These electronic feeders not only provide farmers and researchers with the identification number and the feed intake record of each pig, but also have the capability to record the feeding time, feed intake per visit, and frequency of visits. These data are eventually used to derive different feeding pattern traits or, as termed in several published articles, feeding behavior traits. In the studies of Labroue et al. (1997) [3] and Langdon (2015) [4], it was observed that feeding behavior traits had moderate to high heritability. In addition, earlier researchers reported a favorable genetic correlation between feed intake per meal and rate of feed intake with average daily gain (ADG) and daily feed intake (DFI), and unfavorable genetic correlation with ultrasound backfat thickness (BFT) and lean percentage (LP). Hall (1997) [5] also reported that feed intake per visit, number of visits per day, and time per visit per day were moderately correlated with ADG, BFT, and feed conversion ratio (FCR). Moreover, numerous researchers consistently reported a strong genetic correlation of DFI with feed efficiency traits, including FCR, G:F ratio, and residual feed intake (RFI), and production traits, such as ADG, BFT, and LP [6-8]. Overall, these observations suggest that feeding behaviors or pattern traits respond to selection and may be used as an auxiliary trait in improving feed efficiency (FE) and production traits (PTs) in swine. Given such promising findings, the relationships of feeding pattern traits with FE and PTs remains worthy of investigation, to further verify the earlier findings of previous research. Moreover, there still lack of pertinent data showing the relationship between feeding pattern traits and different measurements of feed efficiency and other production traits, including ADG, ultrasonically measured BFT, LMA, and LP in Duroc pigs (DD). Thus, this study aimed (1) to estimate the genetic parameters of feeding pattern traits, (2) and their genetic relationships to feed efficiency and production traits, (3) and to estimate the non-heritable common spatial pen effect for all studied traits of DD pigs used in this study.

\section{Materials and Methods}

\subsection{Source of Data}

A total of 602 male Duroc (DD) pigs with feeding records at the growing-finishing stage were used in the analyses. These pigs were born from 2018 to 2020 and reared in testing pens of one GGP farm located in South Korea. Each testing pen was equipped with Acemo Genstar (Genstar, Acemo Skiold, Pontivy, France) automatic electronic feeders. The tested pigs included in this study had an initial age (ITA) of $82.99 \pm 1.72$ days and initial weight (IBW) of $43.70 \pm 4.86 \mathrm{~kg}$. All the pigs used in this study were allowed to feed ad libitum for an average of $62.33 \pm 0.65$ days until reaching the average final testing weight (FBW) of $110.23 \pm 10.39 \mathrm{~kg}$.

\subsection{Feed Efficiency and Production Traits}

The feed efficiency traits include the feed conversion ratio (FCR) obtained using the total feed intake and total weight gain during the entire testing period, and five measures of residual feed intake (RFI) obtained by regressing average daily feed intake (ADFI) in models with testing batch (contemporary group, CG) as the common fixed effect and unique covariates for each model including ITA, IBW, ADG for RFI1; ITA, IBW, ADG, BFT for RFI2; ITA, IBW, ADG, LMA for RFI3; ITA, IBW, ADG, BFT and LMA for RFI4; and ITA, ADG, BFT, LMA and average metabolic body weight (AMBW) for RFI5. The IBW was not included in the final model due to its high correlation with AMBW; the latter trait was computed following the formula established by Noblet et al. (1999) [9], with the equation $\left(\mathrm{FBW}^{1.60}-\mathrm{IBW}^{1.60}\right) /\left[1.60\left(\mathrm{FBW}^{1.60}-\mathrm{IBW}^{1.60}\right)\right]$. The production traits 
include on-test average daily gain (ADG), ultrasound backfat thickness (BFT), ultrasound loin muscle area (LMA), and ultrasound lean percentage (LP). BFT, LMA, and LP were all measured at the end of the testing period using A-mode scanners (PIGLOG 105, Frontmatec, Kolding, Denmark). The mean values of feed efficiency and production traits in this study are presented in Table 1.

Table 1. Descriptive statistics of production and feed efficiency trait of Duroc pigs.

\begin{tabular}{ccccccc}
\hline Traits & $\mathbf{N}$ & Unit & Mean & SD & Minimum & Maximum \\
\hline ADG & 603 & g/day & 1067.32 & 129.55 & 642.86 & 1417.74 \\
BFT & 603 & $\mathrm{~mm}$ & 14.23 & 2.26 & 8.00 & 22.00 \\
AMBW & 603 & $\mathrm{~kg} 0.60$ & 13.43 & 0.74 & 11.15 & 15.43 \\
FCR & 602 & $\mathrm{~kg} / \mathrm{kg}$ & 2.30 & 0.19 & 1.68 & 3.13 \\
LMA & 603 & $\mathrm{~cm}^{2}$ & 26.03 & 2.98 & 17.98 & 35.21 \\
LP & 603 & $\%$ & 56.95 & 2.23 & 50.00 & 64.50 \\
RFI1 & 602 & g/day & 0.00 & 167.78 & -536.28 & 885.39 \\
RFI2 & 602 & g/day & 0.00 & 164.59 & -524.75 & 905.40 \\
RFI3 & 602 & g/day & 0.00 & 167.65 & -541.20 & 895.56 \\
RFI4 & 602 & g/day & 0.00 & 164.56 & -527.46 & 910.54 \\
RFI5 & 602 & g/day & 0.00 & 164.60 & -528.84 & 909.22 \\
\hline
\end{tabular}

SD; standard deviation; ADG, on-test average daily gain; BFT, backfat thickness; AMBW, average metabolic body weight; FCR, feed conversion ratio; LMA, loin muscle area; LP, lean percentage; RFI1-RFI model 1; RFI2-RFI model 2; RFI3-RFI model 3; RFI4-RFI model 4; RFI5-RFI model 5; g, grams; mm, millimeter; $\mathrm{kg}$, kilograms; $\mathrm{kg} / \mathrm{kg}$, kilograms feed consumed over kilograms gain in weight; $\mathrm{cm}^{2}$, square centimeter.

\subsection{Electronic Feeder and Criteria Set for Filtering Feeding Visits Records}

The single-place automatic electronic feeders (Acemo Genstar) used by the GGP farm provided pigs with $24 \mathrm{~h}$ feed supply during the entire test duration; however, the feeders only allowed one pig to eat at a time. The feeding and non-feeding visits of individual pigs were measured using an electronic transponder installed in the ear tag of each pig. This transponder sent a radio frequency together with the unique identification number of each pig whenever they entered the electronic feeders.

A total of 367,902 raw feeder visit records were obtained using Acemo Genstar electronic feeders. The raw records comprised the pig identification number from the electronic transponder located in the ear tag of each pig, the time of entering and leaving the feeder, and the feed intake per visit. This initial information obtained from the electronic feeders was used to derive other feeding pattern traits, including the number of visits per day, occupation time inside the feeder per day and per visit, and feeding rate per visit. Subsequently, all the derived feeding pattern traits were filtered using the criteria presented in the studies of Eissen et al. (1998) [10] and Casey et al. (2005) [11]. These criteria include feed intake per visit (FIV) of not $<-20 \mathrm{~g}$ and $>2000 \mathrm{~g}$ or FIV of not $>20 \mathrm{~g}$ if occupation time per visit (OTV) is equal to $0 \mathrm{~s}$; OTV of not $<0 \mathrm{~s}$ and $>3600 \mathrm{~s}$; feeding rate (FRV) per visit of not $>500 \mathrm{~g} / \mathrm{min}$ if FIV $<50 \mathrm{~g}$; FRV of not $>110 \mathrm{~g} / \mathrm{min}$ if FIV was $\geq 50 \mathrm{~g}$, with preceding or following visit having FIV of $<-20 \mathrm{~g}$; FRV of not $>170 \mathrm{~g} / \mathrm{min}$ if FIV was $\geq 50 \mathrm{~g}$ with no preceding or following visit having FIV of $<-20 \mathrm{~g}$; FRV of $=0 \mathrm{~g} / \mathrm{min}$ with OTV of $>500 \mathrm{~s}$; FRV of $<2 \mathrm{~g} / \mathrm{min}$ except for those visits with FRV $=0 \mathrm{~g} / \mathrm{min}$; all visits except the last visit to the feeder during the test period should not have a leading time difference (LTD) and following time difference (FTD) of $<0 \mathrm{~s}$. The related feeder visits that do not fit with these set criteria for each feeding pattern trait were removed from the dataset before the analyses. After filtering, 366,272 feeding pattern records remained.

\subsection{Feeding Pattern Traits}

The filtered feeding and non-feeding records obtained from the electronic feeders were then used to derived the following feeding patterns traits, namely, average number of feeding visits per day (ANVD), calculated by dividing the total number of visits by the number of feeding days; average feed intake per feeding visit (AFIV), calculated by dividing total feed intake by the total number of feeding visits throughout the test duration; 
average occupation time per day (AOTD), calculated by dividing total occupation time inside the feeder by the number of feeding days; average occupation time per visit (AOTV), calculated by dividing total occupation time inside the feeder by the total number of visits; average feed intake rate per day (ADFR), calculated by dividing total feed intake by the total feeding time inside the feeder; average feed intake rate per visit (AFRV), computed by averaging the feed intake rate per feeding visit of each pig throughout the test duration. In addition, average daily feed intake (ADFI) was calculated by dividing total feed intake by the number of feeding days.

\subsection{Statistical Analyses}

Prior to the analyses of genetic parameters, the potential fixed effects, including the testing batch as a contemporary group ( $C G=22$ levels), parity of dam, testing days, potential fixed covariates including ITA and IBW for ADG, FCR, RFI, and all feeding pattern traits, and FTA and FBW for BFT, LMA, and LP, were initially tested for their significance using the PROC GLM procedure of the SAS package/PC version 9.4 [12]. Among the tested fixed effects, only CG was found to be significant in most traits, whereas IBW was found to be significant as a fixed covariate for ADG, FCR, ADFI, AOTD, ADFR, AFRV, and AFIV, and FBW was found to be significant for BFT, LMA, and LP. CG was found to be insignificant in all RFI measures due to the initial inclusion of this fixed effect in the estimation of their value. However, CG was still included as a fixed effect for all RFI measures, similar to the studies of Lu et al. (2017) [7], Gilbert et al. (2007) [13], and Saintilan et al. (2013) [14].

The genetic parameters and (co)variance components for each feeding pattern trait were estimated using three animal models and the restricted maximum likelihood method (REML) of the WOMBAT program [15]. The three animal models used in the analyses are presented below:

$$
\begin{gathered}
\text { Model 1: } \mathrm{y}_{\mathrm{t}}=\mathrm{Xb}+\mathrm{Z}_{1} \mathrm{a}+\mathrm{Z}_{2} \mathrm{c}+\mathrm{e} \\
\text { Model 2: } \mathrm{y}_{\mathrm{t}}=\mathrm{Xb}+\mathrm{Z}_{1} \mathrm{a}+\mathrm{Z}_{3} \mathrm{p}+\mathrm{e} \\
\text { Model 3: } \mathrm{y}_{\mathrm{t}}=\mathrm{Xb}+\mathrm{Z}_{1} \mathrm{a}+\mathrm{Z}_{2} \mathrm{c}+\mathrm{Z}_{3} \mathrm{p}+\mathrm{e}
\end{gathered}
$$

where $y_{t}$ is the vector of observations for each feeding pattern trait; $b$ is the vector of fixed effects of the contemporary group (CG or testing batch $=22$ levels); $a$ is the vector of additive genetic effects; $c$ is the vector for the common litter effect; $p$ is the vector for random common spatial pen effects; $\mathrm{e}$ is the vector for random residual effects. $X, Z_{1}, Z_{2}$, and $Z_{3}$ are the corresponding indices matrices for fixed, additive genetic, common litter, and common spatial pen effects, respectively. The animal genetic, common litter, and spatial pen terms were assumed to be independent and distributed as a $\sim \mathrm{N}\left(0, \mathrm{~A} \sigma_{\mathrm{a}}^{2}\right), \mathrm{c} \sim \mathrm{N}\left(0, \mathrm{I}_{\mathrm{c}}^{2}\right), \mathrm{p} \sim \mathrm{N}\left(0, \operatorname{I\sigma }_{\mathrm{p}}^{2}\right)$, and $\mathrm{e} \sim \mathrm{N}\left(0, \mathrm{I} \sigma_{\mathrm{e}}^{2}\right)$, respectively. $\mathrm{A}, \mathrm{I}, \sigma_{\mathrm{a}}^{2}, \sigma_{\mathrm{c}}^{2}, \sigma_{\mathrm{p}}^{2}$, and $\sigma_{\mathrm{e}}^{2}$ represent the numerator relationship matrix (NRM), identity matrix, variances of random animal genetic, random common litter effect, random common spatial pen effect, and random residual error effect, respectively. A total of 328 litters and 20,226 animals in the pedigree were included in the analyses.

The heritability estimate $\left(h^{2}\right)$ was obtained as the ratio of additive genetic $\left(\sigma_{a}^{2}\right)$ to phenotypic $\left(\sigma_{\mathrm{p}}^{2}\right)$ variance as shown in the equation below:

$$
\mathrm{h}^{2}=\frac{\sigma_{\mathrm{a}}^{2}}{\sigma_{\mathrm{p}}^{2}}
$$

Bivariate analyses were undertaken to estimate the genetic and phenotypic correlation of feeding pattern traits to feed efficiency and production traits. The animal model 1, including the corresponding covariates for each trait, was used for these analyses. 


\subsection{Model Comparison}

The basis of comparison among the models in this study was undertaken using the likelihood ratio test (LRT). The LRT was applied to test the significance between the full and nested models [16]. The equation used to compute the LRT value is shown below:

$$
\mathrm{LRT}=(-2 \log L \text { from nested model })-(-2 \log L \text { from full model })
$$

where $\log L$ is the logarithm of the restricted maximum likelihood function. The statistical significance for the LRT value obtained in this study was set at $p<0.05$. The obtained LRT value was compared to the chi-square $\left(\mathrm{X}^{2}\right)$ distribution based on the degrees of freedom derived from subtracting the number of parameters in two models. A significant LRT value indicates that the full model has better model fit than the nested model, whereas an insignificant LRT value indicates that the nested model with fewer parameters is the most appropriate model.

\section{Results and Discussion}

\subsection{Descriptive Statistics of Feeding Pattern Traits}

The descriptive statistics for feeding pattern traits are presented in Table 2. The mean values for ADFI, AOTD, AOTV, ADFR, AFRV, ANVD, and AFIV were $2.48 \mathrm{~kg} / \mathrm{day}$, $81.26 \mathrm{~min} /$ day, $8.85 \mathrm{~min} /$ visit, $31.86 \mathrm{~g} / \mathrm{min}, 28.71 \mathrm{~g} / \mathrm{min}, 9.93$ visits/day, and $297.06 \mathrm{~g} /$ day, respectively. Using the electronic feeders manufactured by the same manufacturer (ACEMA 64), Do et al. (2013) [6] reported that DD boars with body weight ranging from 30 to $100 \mathrm{~kg}$ had comparable mean values for daily feed intake (DFI), time per day (TPD), time per visit (TPV), feed intake rate (FR), number of visits to feeder per day (NVD), and feed intake per visit (FPV) of $2.40 \mathrm{~kg} /$ day, $81.05 \mathrm{~min} /$ day, $8.58 \mathrm{~min} / \mathrm{visit}, 30.61 \mathrm{~g} / \mathrm{min}$, 11.07 visits/day, and $250 \mathrm{~g} /$ visit, respectively. Moreover, the same researchers reported that DD had higher TPD and NVD than LL (Landrace) and Yorkshire (YY) boars. Using FIRE feeders, Lu et al. (2017) reported lower mean ADFI (2.15 kg), AOTD (61.92 min), and ANVD (5.77 visits/day), and higher mean ADFR ( $36.72 \mathrm{~g} / \mathrm{min}), \operatorname{AFRV}(36.72 \mathrm{~g} / \mathrm{min})$, AFIV (471.62 g/visit), and AOTV (12.97 min/visit) in DD boars.

Table 2. Descriptive statistics for feeding pattern traits of Duroc pigs.

\begin{tabular}{cccccc}
\hline Traits & Unit & Mean & SD & Minimum & Maximum \\
\hline ADFI & kg/day & 2.48 & 0.34 & 1.62 & 3.86 \\
AOTD & min/day & 81.26 & 14.14 & 47.43 & 134.23 \\
AOTV & min/visit & 8.85 & 2.73 & 3.29 & 18.80 \\
ADFR & g/min & 31.86 & 6.30 & 16.52 & 50.85 \\
AFRV & g/min & 28.71 & 6.05 & 15.30 & 46.57 \\
ANVD & visits/day & 9.93 & 3.16 & 3.79 & 25.23 \\
AFIV & g/visit & 297.06 & 96.44 & 89.83 & 826.76 \\
\hline
\end{tabular}

ADFI, average daily feed intake; AOTD, average occupation time per day; ADFR, average daily feeding rate; ANVD, average number of visits per day; AFIV, average feed intake per feeding visit; AOTV, average occupation time per visit; AFRV, average feeding rate per feeding visit; SD, standard deviation; kg, kilograms; min, minutes; g, grams.

Compared to observed mean values for DD in this study, Labroue et al. (1997) [3] reported lower DFI (2.19 kg/day and $2.25 \mathrm{~kg} /$ day) and TPD (58.90 min and $59.10 \mathrm{~min})$, and higher FR (39.90 g/min and $41.50 \mathrm{~g} / \mathrm{min})$ in Large White (LW) and LL pigs, respectively, using electronic feeder manufactured by the same manufacturer but with a different model (ACEMA 48). Von Felde et al. (1996) [17] also reported that LW and LL had lower mean values for TPD (49.20 $\mathrm{min}$ ) and DFI (2.36 kg/day), and much higher mean values for TPV (11.54 min/visit), FPV (565.80 g/visit), and FR ( $49 \mathrm{~g} / \mathrm{min})$, in five testing periods using ACEMA 48 feeders.

However, there were a number of differences with our study and these cited references, including on-test growth rate of the pigs, the calculation methods for some feeding pattern 
traits, the starting weights and testing duration, and the numbers of pigs per pen. These studies also housed mixed breeds in the same testing pen. According to Do et al. (2013) [6], the use of DD, LL, and YY pigs, which grow faster and eat more compared to pigs used in previous studies, may explain the higher mean TPD observed in their study. Moreover, Labroue et al. (1994) [18] reported that mixing of LW and LR in a similar testing pen could influence the feeding pattern of the latter breed. Based on these observations, the differences in the observed mean values may be due to various factors, including the differences in the growth rate of the tested pigs, breed differences, differences in electronic feeder, discrepancies in the initial testing weight and testing period, variations in the calculation method for feeding pattern traits, the number of pigs allowed for each testing pen, and the housing of mixed breeds in the same testing pen.

\subsection{Heritability Estimates of Feeding Pattern Traits}

The heritability estimates for feeding pattern traits are presented in Table 3 . The lowest heritability estimates among feeding pattern traits of DD were observed for models 1 and 3 of ADFI (0.20 and 0.19) and AFIV (0.18 and 0.19). However, the same traits had moderate heritability for model 2. ANVD had moderate heritability, ranging from 0.27 to 0.34 , with model 2 having the highest estimate. The observed low heritability for model 1 in ADFI was consistent with the finding of Lu et al. (2017) [7], with reported heritability of 0.18 using a model with random animal effects. However, similar studies reported lower heritability for a model with a random spatial pen effect. In contrast, numerous researchers reported moderate to high heritability estimates of 0.36 [4], 0.41 [6], 0.44 [19], and 0.49 [20] for ADFI in DD pigs. The observed heritability for AFIV and ANVD in this study were far lower than those observed, respectively, by Langdon (2015) [4] of 0.66 and 0.53; Do et al. (2013) [6] of 0.49 and 0.44; and Lu et al. (2017) [7], ranging from 0.37 to 0.49 and 0.36 to 0.68 for the same pig breed. Moreover, Von Felde et al. (1996) [17] reported low to moderate heritability for feed intake per day ( 0.16 to 0.30 ), and high heritability estimates for number of visits per day (0.43) and feed intake per visit (0.51) in LW and LL breeds.

The time of stay inside the feeder trait in this study includes both AOTD and AOTV. Estimates of heritability for AOTD in this study were found to be identical for all models (0.73) due to almost zero litter and pen effect for this trait. Such high heritability estimates for AOTD were also observed in DD boars by Do et al. (2013) [6], of 0.56, and Lu et al. (2017) [7], of 0.50 to 0.71. However, Langdon (2015) [4] reported much lower heritability of 0.22 for the same breed and trait. In AOTV, all models had moderate heritability ranging from 0.21 to 0.31 . Contrary to this observation, several researchers reported much higher heritability for time per visit of 0.88 [4], 0.47 [6], and 0.51 to 0.58 [7] for the same pig breed. Von Felde et al. (1996) [17] and Kavlak and Uimari (2019) [8] also reported moderate to high heritability for both time traits of LW and LL, and Yorkshire (YY), respectively.

In terms of feeding rate traits, including ADFR and AFRV, model 2 had higher estimates than models 1 and 3. Specifically, high heritability estimates were found for both feeding rate traits ranging from 0.59 to 0.64 . Such high heritability for feeding rate traits was also observed by Do et al. (2013) [6], of 0.56, and Langdon (2015) [4], of 0.76, whereas Lu et al. (2017) [7] reported lower heritability estimates for feeding rate traits ranging from 0.19 to 0.35 for ADFR and 0.18 to 0.48 for AFRV of the same pig breed. Von Felde et al. (1996) [17] and Labroue et al. (1997) [3] also reported high heritability for the rate of feed intake in LW and LL; however, these authors still observed lower heritability for the latter trait than that observed in this study. 
Table 3. Heritability estimates and variance components for feeding pattern traits of Duroc pigs.

\begin{tabular}{|c|c|c|c|c|c|c|c|c|c|c|c|c|c|c|c|c|c|c|}
\hline \multirow{2}{*}{ Traits } & \multicolumn{6}{|c|}{ Model 1} & \multicolumn{6}{|c|}{ Model 2} & \multicolumn{6}{|c|}{ Model 3} \\
\hline & $\sigma_{a}^{2}$ & $\sigma_{e}^{2}$ & $h^{2}$ & $c^{2}$ & $-2 \log L$ & LRT1 & $\sigma_{a}^{2}$ & $\sigma_{e}^{2}$ & $h^{2}$ & $\mathrm{p}^{2}$ & $-2 \log L$ & LRT2 & $\sigma_{a}^{2}$ & $\sigma_{e}^{2}$ & $h^{2}$ & $c^{2}$ & $p^{2}$ & $-2 \log L$ \\
\hline ADFI & $1.58 \times 10^{-2}$ & $5.14 \times 10^{-2}$ & $0.20 \pm 0.11$ & 0.14 & -860.73 & $36.02 *$ & $2.60 \times 10^{-2}$ & $4.60 \times 10^{-2}$ & $0.32 \pm 0.10$ & 0.13 & -890.74 & $6.01 *$ & $1.52 \times 10^{-2}$ & $4.41 \times 10^{-2}$ & $0.19 \pm 0.10$ & 0.14 & 0.13 & -896.75 \\
\hline AOTD & 129.58 & 47.66 & $0.73 \pm 0.12$ & 0.00 & 3511.55 & $0.06^{\mathrm{ns}}$ & 130.13 & 46.97 & $0.73 \pm 0.11$ & 0.003 & 3511.49 & $0.01^{\mathrm{ns}}$ & 129.58 & 46.79 & $0.73 \pm 0.12$ & 0.004 & 0.003 & 3511.49 \\
\hline AOTV & 1.62 & 4.50 & $0.25 \pm 0.12$ & 0.07 & 1703.41 & $39.13 *$ & 2.17 & 3.88 & $0.31 \pm 0.11$ & 0.13 & 1666.57 & $2.29^{\mathrm{ns}}$ & 1.46 & 3.89 & $0.21 \pm 0.11$ & 0.08 & 0.14 & 1664.28 \\
\hline ADFR & 19.07 & 11.60 & $0.62 \pm 0.11$ & 0.00 & 2526.79 & 23.19 * & 20.45 & 8.95 & $0.64 \pm 0.10$ & 0.08 & 2503.62 & $0.02^{\mathrm{ns}}$ & 20.28 & 8.89 & $0.63 \pm 0.11$ & 0.01 & 0.09 & 2503.60 \\
\hline AFRV & 17.59 & 12.11 & $0.59 \pm 0.12$ & 0.00 & 2515.70 & $61.95 *$ & 20.42 & 6.84 & $0.63 \pm 0.11$ & 0.17 & 2453.75 & $0.00^{\mathrm{ns}}$ & 20.42 & 6.84 & $0.63 \pm 0.11$ & 0.00 & 0.17 & 2453.75 \\
\hline ANVD & 2.80 & 6.17 & $0.30 \pm 0.12$ & 0.04 & 1899.44 & 42.05 * & 3.42 & 5.10 & $0.34 \pm 0.10$ & 0.15 & 1858.96 & $1.57^{\mathrm{ns}}$ & 2.72 & 5.05 & $0.27 \pm 0.11$ & 0.07 & 0.15 & 1857.39 \\
\hline AFIV & 1437.74 & 5630.88 & $0.18 \pm 0.11$ & 0.11 & 5823.03 & $77.25 *$ & 2954.99 & 4016.33 & $0.33 \pm 0.11$ & 0.22 & 5750.02 & 4.24 * & 1654.72 & 4107.01 & $0.19 \pm 0.10$ & 0.11 & 0.23 & 5745.78 \\
\hline
\end{tabular}

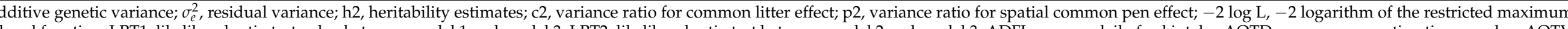
2, likelihood ratio test between model 2 and model 3; ADFI, average daily feed intake; AOTD, average occupation time per day; AOTV

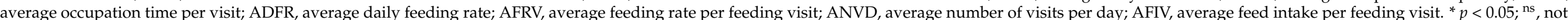
significant. 
The observed differences for the heritability estimate of feeding pattern traits may be attributed to differences in breed, sex, feeder and pen design, pen density, number of tested animals, calculation differences for each feeding pattern trait, and model differences in the estimation of genetic parameters. According to Do et al. (2013) [6], a possible bias in the estimate of heritability and genetic parameters may arise when data are from selected pigs, such as a sample of mostly males with high genetic merit is tested. This observation may be relevant because only candidate DD boars were included in this study. Despite these observed differences in heritability estimates, the moderate to high heritability for majority feeding pattern traits obtained in this study suggests a rapid improvement in these traits once they are considered in the breeding program. However, to avoid a negative impact on the performance of the swine industry, familiarization with the genetic correlation of these feeding pattern traits to feed efficiency and production traits must always be considered before including them in the breeding program.

\subsection{Additive Common Litter Effect}

The common litter effect accounted for nil to a small portion of the total variability among feeding pattern traits in DD pigs, with corresponding percentage values of $0.00 \%$ to $14.00 \%$ (Table 3). The percentage of variance explained by the litter effect for ADFI, AOTD, AOTV, ADFR, AFRV, ANVD, and AFIV in model 1 was $14.00 \%, 0.00 \%, 7.00 \%, 0.00 \%, 0.00 \%$, $4.00 \%$ and $11.00 \%$, respectively. The corresponding percentage value for similar feeding pattern traits using model 3 was $14.00 \%, 0.40 \%, 8.00 \%, 1.00 \%, 0.00 \%, 7.00 \%$, and $11.00 \%$, respectively. These findings were comparable to those reported by Hall et al. (1997) [5], with percentage variance due to a common litter effect of $12.00 \%, 0.00 \%, 0.00 \%, 0.00 \%$, $5.00 \%$, and $5.00 \%$ for daily feed intake, time per day, time per visit, feeding rate, number of visits per day, and feed intake per visit, respectively. The noticeable small percentage of variance due to the litter effect in this study indicates that the addition of the common litter effect would likely result in small improvement in the precision of breeding value estimation for most feeding pattern traits of DD pigs.

\subsection{Additive Common Spatial Pen Effect}

The common spatial pen effect in models 2 and 3 in this study accounted for $0.30 \%$ to $23.00 \%$ of the total variability of feeding pattern traits (Table 3). The highest percentage variance due to the common pen effect was observed in AFIV, whereas the lowest was observed in AOTD. In the study of Do et al. (2013) [6], a substantial percentage variance due to common pen effects was also observed in some feeding pattern traits for the same pig breed with corresponding estimates of $4.00 \%$ for DFI, 3.00\% for TPD, $12.00 \%$ for TPV, $5.00 \%$ for FR, $9.00 \%$ for NVD, and $10.00 \%$ for FPV. It should be noted, however, that the specific pen effects (physical pen location, social pen mate interaction) used by the latter researchers were not clearly mentioned in their study. Overall, these observations indicate that the inclusion of the common spatial pen effect may result in a substantial improvement in the estimation of genetic parameters for most feeding pattern traits of DD pigs. However, testing the goodness of fit when adding additional random effects must be considered to determine the best model fit that would result in the most precise estimates of breeding value for these traits.

\subsection{Model Comparison}

The likelihood ratio test among models for each trait was used as a basis for model comparison (Table 3). In all feeding pattern traits, with the exception of AOTD, model 3 was found to be the more appropriate model compared to model 1 by having significant $(p<0.05)$ LRT1 values ranging from 23.19 to 77.25 . The LRT1 value for AOTD, of 0.06, was found to be lower than the $X^{2}$ distribution, which indicates that model 1 with fewer parameters was the most appropriate for this trait. The feeding pattern traits, with the exception of ADFI and AFIV, had insignificant $(p>0.05)$ LRT2 values ranging from 0.00 to 1.57 , which indicates that model 2 was more appropriate than model 3 in most feeding pattern traits. 


\subsection{Correlation among Feeding Pattern Traits}

The genetic and phenotypic correlation among feeding pattern is presented in Table 4 . The ADFI in DD had low positive genetic and phenotypic correlation with AOTD (0.23 and 0.22); close to zero genetic and moderate phenotypic correlation with ADFR (0.12 and 0.42 ) and AFRV (0.12 and 0.39); high and positive genetic, and close to zero phenotypic correlation with ANVD (0.52 and 0.07); and close to zero genetic and low positive phenotypic correlation with AFIV ( -0.06 and 0.26$)$. In contrast, ADFI had high negative genetic and zero phenotypic correlation with AOTV ( -0.60 and 0.00$)$. These observations indicate that any increase in ADFI would result in DD pigs eating for a longer time per day, spending less time inside the feeder per visit, and making more frequent visits to the feeder. Most of these observations were in the same direction as the findings of Do et al. (2013) [6], but with different levels of magnitude. Specifically, daily feed intake (DFI) had low to moderate genetic and phenotypic correlation with time per day (TPD), number of visits per day (NVD), feeding rate (FR), feeding per visit (FPV), and time per visit (TPV), ranging from 0.19 to 0.32 and 0.16 to 0.46 , respectively, whereas DFI had low negative genetic correlation $(-0.16)$ and close to zero phenotypic correlation (-0.09) to TPV. Lu et al. 2017 [7] also reported that ADFI had moderate to high positive genetic correlation with all feeding behavior traits, including AOTD, ANVD, ADFR, AFRV, and AFIV, with correlation coefficients ranging from 0.47 to 0.83 . However, the latter researchers reported moderate positive genetic correlation between ADFI and AOTV (0.42), which was opposite with that observed in this study.

Table 4. Genetic (below diagonal) and phenotypic (above diagonal) correlation among feeding pattern traits in Duroc pigs.

\begin{tabular}{cccccccc}
\hline Traits & ADFI & AOTD & AOTV & ADFR & AFRV & ANVD & AFIV \\
\hline ADFI & & 0.22 & 0.00 & 0.42 & 0.39 & 0.07 & 0.26 \\
AOTD & 0.23 & & 0.23 & -0.76 & -0.73 & 0.33 & -0.19 \\
AOTV & -0.60 & 0.41 & & -0.22 & -0.03 & -0.76 & 0.81 \\
ADFR & 0.12 & -0.94 & -0.45 & & 0.94 & -0.24 & 0.33 \\
AFRV & 0.12 & -0.92 & -0.38 & 0.99 & & -0.42 & 0.49 \\
ANVD & 0.52 & 0.56 & -0.50 & -0.43 & -0.48 & & -0.83 \\
AFIV & -0.06 & -0.65 & 0.33 & 0.66 & 0.70 & -0.88 & \\
\hline
\end{tabular}

ADFI, average daily feed intake; AOTD, average occupation time per day; AOTV; average occupation time per visit; ADFR, average daily feeding rate; AFRV, average feeding rate per visit; ANVD, average number of visits per day; AFIV, average feed intake per visit.

A moderate genetic and low phenotypic correlation between AOTD and AOTV (0.41 and 0.23 ) was observed in this study. The AOTD had high negative genetic and phenotypic correlation with ADFR ( -0.94 and -0.76$)$ and AFRV ( -0.92 and -0.73$)$, whereas AOTV had moderate negative genetic and low phenotypic correlation with ADFR $(-0.45$ and $-0.22)$, and moderate negative genetic and insignificantly different from zero phenotypic correlation with AFRV ( -0.38 and 0.03 ). Moreover, the AOTD had high positive genetic and moderate positive phenotypic correlation with ANVD (0.56 and 0.33), and high negative genetic and low negative phenotypic correlation with AFIV ( -0.65 and -0.19$)$. Conversely, AOTV had high negative genetic and phenotypic correlation with ANVD ( -0.50 and $-0.76)$, and moderate genetic and high phenotypic correlation with AFIV (0.33 and 0.81). The same correlation in terms of direction but with different magnitude for similar traits of DD pigs was observed in the study of Do et al. (2013) [6]. In particular, TPD had positive low genetic ( 0.24$)$ and phenotypic correlation $(0.23)$ with TPV, and high negative genetic and phenotypic correlation with FR $(-0.87$ and -0.78$)$, whereas TPV had moderate negative genetic and low phenotypic correlation with FR $(-0.30$ and -0.19$)$. The same researchers also observed a low positive genetic and phenotypic correlation between TPD and NVD (0.19 and 0.15), and negative low genetic and phenotypic correlation between TPD and FPV $(-0.16$ and -0.12$)$, whereas TPV had high negative genetic and phenotypic correlation with NVD ( -0.87 and -0.76$)$ and high positive genetic and phenotypic correlation with 
FPV (0.88 and 0.87). The observed contradicting relationships between the length of time spent inside the feeder traits and feeding rate traits were expected because the former traits were used as the basis for computing the feeding rate traits, which implies that the feeding rate of DD pigs will increase and decrease when pigs spend a shorter and longer time inside the feeder, respectively. The remaining observations indicate that selection for DD pigs with shorter AOTD and longer AOTV would likely result in fewer visits to the feeder, and higher feed intake per visit, whereas the opposite is true for longer AOTD and shorter AOTV.

In the remaining feeding pattern traits, a varied relationship was observed. The ANVD had low to high negative genetic and phenotypic correlation with ADFR ( -0.43 and $-0.24)$ and AFRV ( -0.48 and -0.42$)$. The AFIV had high positive genetic and moderate phenotypic correlation with ADFR (0.66 and 0.33), and high positive genetic and moderate phenotypic correlation with AFRV (0.70 and 0.49). These observations were similar in direction but with far higher magnitude than those observed by Do et al. (2013) [6], who reported insignificantly different from zero genetic and phenotypic correlation between NVD and FR ( -0.04 and -0.04 , respectively), and close to zero genetic and phenotypic correlation between FPV and FR (0.10 and 0.13, respectively). Lu et al. (2017) [7] also reported a negative genetic correlation between ANVD and ADFR (-0.28) and between ANVD and AFRV (-0.33), and a high and positive genetic correlation between AFIV and ADFR (0.63) and between AFIV and AFRV (0.63). These observed correlations simply indicate that DD pigs with a faster feeding rate would result in fewer visits to the feeder and lower feed intake per visit, whereas the opposite is true for DD pigs with a slower feeding rate.

In general, these findings indicate that the majority of the feeding pattern traits were genetically and phenotypically correlated with each other. Among the correlated feeding pattern traits, the most interesting finding is the negative relationship between the time spent inside the feeder traits and the feeding rate traits, which indicates that selection for one of these traits would result in a negative change to other traits, and eventually in the production and feed efficiency traits to which they are correlated. Therefore, extra caution should be applied, and familiarization with the relationship between feeding pattern traits should always be considered, before including them in the breeding program as a supplementary trait to improve the feed efficiency and production performance of pigs.

\subsection{Correlation between Feeding Pattern and Production Traits in Duroc pigs}

The genetic and phenotypic correlation between feeding patterns and production traits in DD pigs is presented in Table 5. The ADFI had high positive genetic correlation and moderate to high phenotypic correlation with on-test ADG (0.73 and 0.78), BFT (0.60 and 0.36$)$, FCR (0.54 and 0.45), and all RFI measures ( 0.67 to 0.72 and 0.61 to 0.63$)$. Moreover, ADFI was found to have a high negative genetic correlation of -0.58 and -0.74 , and close to zero and moderate negative phenotypic correlation of -0.07 and -0.33 to LMA and LP, respectively. In similar pig breed, Do et al. (2013) [6] also reported that ADFI had moderate to high positive genetic and phenotypic correlation, respectively, with on-test ADG (0.45 and 0.32), BFT (0.29 and 0.34), FCR (0.67 and 0.65), RFI1 (0.95 and 0.90), and RFI2 (0.88 and 0.91). Lu et al. (2017) [7] also observed that ADFI had high positive genetic correlation with on-test ADG (0.82), BFT (0.71), and RFI6 (0.88) in the same pig breed. 
Table 5. Genetic $\left(\mathbf{r}_{\mathbf{g}}\right)$ and phenotypic correlation $\left(\mathbf{r}_{\mathbf{p}}\right)$ of feed efficiency traits with production traits and feed intake of Duroc pigs.

\begin{tabular}{|c|c|c|c|c|c|c|c|c|c|c|c|c|c|c|c|}
\hline \multirow{2}{*}{ Category } & \multirow{2}{*}{ Traits } & \multicolumn{2}{|c|}{ ADFI } & \multicolumn{2}{|c|}{ AOTD } & \multicolumn{2}{|c|}{ AOTV } & \multicolumn{2}{|c|}{ ADFR } & \multicolumn{2}{|c|}{ AFRV } & \multicolumn{2}{|c|}{ ANVD } & \multicolumn{2}{|c|}{ AFIV } \\
\hline & & $r_{g}$ & $\mathbf{r}_{\mathrm{p}}$ & $r_{g}$ & $r_{p}$ & $r_{g}$ & $r_{p}$ & $r_{g}$ & $r_{p}$ & $\mathbf{r}_{\mathrm{g}}$ & $r_{p}$ & $r_{g}$ & $r_{p}$ & $r_{g}$ & $r_{p}$ \\
\hline \multirow{4}{*}{ Production } & ADG & 0.73 & 0.78 & 0.04 & 0.16 & -0.63 & 0.00 & 0.21 & 0.33 & 0.28 & 0.31 & 0.35 & 0.06 & 0.02 & 0.21 \\
\hline & BFT & 0.60 & 0.36 & 0.26 & 0.10 & 0.38 & 0.10 & 0.04 & 0.10 & -0.02 & 0.09 & 0.18 & 0.01 & 0.24 & 0.12 \\
\hline & LMA & -0.58 & -0.07 & -0.05 & -0.01 & 0.00 & 0.03 & -0.05 & -0.02 & -0.01 & -0.03 & -0.02 & -0.03 & -0.02 & -0.01 \\
\hline & LP & -0.74 & -0.33 & -0.39 & -0.11 & 0.12 & -0.05 & 0.06 & -0.05 & 0.16 & -0.05 & -0.77 & -0.05 & 0.26 & -0.08 \\
\hline \multirow{6}{*}{$\begin{array}{l}\text { Feed Ef- } \\
\text { ficiency }\end{array}$} & FCR & 0.54 & 0.45 & 0.27 & 0.12 & -0.17 & 0.00 & -0.01 & 0.17 & -0.08 & 0.16 & 0.34 & 0.03 & -0.13 & 0.11 \\
\hline & RFI1 & 0.72 & 0.63 & 0.33 & 0.15 & -0.25 & 0.00 & -0.06 & 0.25 & -0.13 & 0.23 & 0.46 & 0.04 & -0.16 & 0.14 \\
\hline & RFI2 & 0.68 & 0.61 & 0.24 & 0.14 & -0.40 & -0.02 & 0.00 & 0.26 & -0.04 & 0.23 & 0.38 & 0.04 & -0.13 & 0.13 \\
\hline & RFI3 & 0.71 & 0.63 & 0.33 & 0.15 & -0.26 & 0.00 & -0.06 & 0.25 & -0.12 & 0.23 & 0.47 & 0.04 & -0.16 & 0.14 \\
\hline & RFI4 & 0.67 & 0.61 & 0.24 & 0.14 & -0.41 & -0.02 & 0.00 & 0.26 & -0.04 & 0.23 & 0.39 & 0.04 & -0.13 & 0.13 \\
\hline & RFI5 & 0.67 & 0.61 & 0.24 & 0.14 & -0.41 & -0.02 & 0.00 & 0.26 & -0.04 & 0.23 & 0.39 & 0.04 & -0.13 & 0.13 \\
\hline
\end{tabular}

ADFI, average daily feed intake; AOTD, average occupation time per day; AOTV; average occupation time per visit; ADFR, average daily feeding rate AFRV, average feeding rate per visit; ANVD, average number of visits per day; AFIV, average feed intake per visit; ADG, on-test average daily gain in weight; BFT, ultrasound backfat thickness; LMA, ultrasound loin muscle, LP, ultrasound lean percentage; FCR, feed conversion ratio; RFI1-RFI model 1; RFI2-RFI model 2; RFI3-RFI model 3; RFI4-RFI model 4; RFI5-RFI model 5.

The genetic correlation of AOTD and ANVD with most of the production and feed efficiency traits ranged from low to moderate. Specifically, the AOTD and ANVD had low to moderate positive genetic correlation to BFT (0.26 and 0.18), FCR (0.27 and 0.34), RFI1 (0.33 and 0.46), RFI2 (0.24 and 0.38), RFI3 (0.33 and 0.47), RFI4 (0.24 and 0.39) and RFI5 (0.24 and 0.39), respectively. Contrastingly, AOTD and ANVD had moderate and high negative genetic correlations to LP ( -0.39 and -0.77 , respectively). Moreover, the AOTD was found to have no genetic correlation to ADG (0.04), whereas ANVD, ADFR, and AFRV had low to moderate positive genetic correlation to on-test ADG of $0.35,0.20$, and 0.28 , respectively. These observations were in a similar direction but with different magnitude to those observed by Do et al. (2013) [6], where TPD had low positive genetic correlation with on-test ADG (0.23), BFT (0.10), FCR (0.11), RFI1 (0.25), and RFI2 (0.20), whereas NVD had a moderate to high positive genetic relationship to FCR (0.50), RFI1 (0.40), and RFI2 (0.44). Lu et al. (2017) [7] reported similar findings but also found different magnitudes for most of the correlations mentioned earlier, where AOTD had close to zero genetic correlation with on-test ADG (0.11), high and positive genetic correlation with RFI (0.72), and high negative genetic correlation to G:F ratio $(-0.65)$, which is equivalent to a positive correlation coefficient to FCR. Furthermore, the latter researchers also observed a moderate to high positive genetic correlation between feeding rate traits (ADFR and AFRV) and on-test ADG of 0.57 and 0.23 , respectively, and moderate positive genetic correlation between ANVD and RFI (0.23). However, Do et al. (2013) [6] reported a low positive genetic correlation between TPD and on-test ADG (0.23), Lu et al. (2017) [7] reported a low negative genetic correlation between ANVD and BFT (-0.25), and both researchers reported a moderate negative genetic correlation between the number of visits per day and on-test ADG ( -0.26 and -0.28 , respectively), which were all opposite to those observed in this study. The corresponding phenotypic correlations of most genetic correlations discussed above were low, with the exception of the phenotypic correlation between ADFI and most of the production and feed efficiency traits in this study. The low phenotypic correlation of most feeding pattern traits to feed efficiency and production traits is probably due to their low residual or environmental correlation. According to Cheverud (1984) [21], the similarity between genetic and phenotypic correlation occurs when environmental effects act in the same direction and pathways as genetic effects.

These earlier observations suggest DD pigs that eat more per day (ADFI), visit the feeders for a longer period per day (AOTD), and more frequently visit the feeder per day (ANVD) may result in slightly higher BFT, less lean percentage (LP), and less feed efficient pigs due to higher FCR and RFI. According to Rauw et al. (2006) [22], DD barrows that spent longer inside the feeder ate significantly more and had higher RFI. By comparison, the DD pigs in this study, which visited the feeders more frequently (ANVD), may result in faster growing pigs. However, this latter observation was opposite to those observed by Do et al. (2013) [6], Langdon (2015) [4], and Lu et al. (2017) [7], which may 
partly be attributed to the observed high and negative genetic correlation of AOTV with ADG (-0.63), ANVD (-0.50), and ADFI (-0.60), and high and positive genetic correlation between ANVD and ADFI (0.52) in this study (shown in Table 4). These assumptions appeared because any decrease in AOTV may result in higher ADFI and ANVD and, eventually, higher on-test ADG.

In addition to the negative correlation between AOTV and ADG, the earlier trait also had a low to moderate negative genetic correlation with FCR $(-0.17)$, and all RFI measures $(-0.25$ to -0.41$)$, and a moderate positive genetic correlation with BFT (0.38). Most of these observations were in agreement with those reported by Do et al. (2013) [6], where TPV had a low to moderate negative genetic correlation with FCR $(-0.43)$, and RFI $(-0.28$ to -0.31$)$, and a moderate positive genetic correlation with BFT (0.26). However, the same researchers also reported a moderate positive genetic correlation between TPV and on-test ADG (0.35), which was opposite with that observed in this study. The negative genetic correlation between AOTV and ADG in this study may be partly attributed to the observed a moderate to high negative genetic correlation of AOTV with ADFI ( -0.60$)$, AFRV (-0.38), and ANVD (-0.50), and a moderate positive genetic correlation between ANVD and ADG, and AFRV and ADG (0.28), as shown in Table 4. These assumptions appeared because the negative correlation between AOTV and ADFI indicates that pigs spending longer inside the feeder per visit would result in lower ADFI and, eventually, in lower ADG, whereas the association between ANVD and AFRV emerged because any decrease in either of these traits would lead to longer AOTV, which would also result in lower ADFI and ADG. Therefore, the observed genetic relationships of AOTV with production and feed efficiency traits suggest that DD pigs with a longer time spent inside the feeder per visit would result in a lower ADG, less residual feed intake, and higher BFT, whereas the opposite is true for a shorter time spent inside the feeder per visit.

The AFIV had a low positive genetic correlation with BFT (0.24) and LP (0.26). Do et al. (2013) [6] and $\mathrm{Lu}$ et al. (2017) [7] also reported a low positive genetic correlation between feed intake per visit and BFT of 0.29 and 0.15 , respectively. However, both references reported a high genetic correlation between feed intake per visit and on-test ADG ( 0.40 and 0.54 , respectively), which were not found to be correlated in this study. These findings indicate that, as the AFIV increases, there would be a corresponding increase in BFT and LP in DD pigs, whereas the opposite is true when AFIV decreases.

Overall, the most interesting genetic correlation was observed between AOTD and most production and feed efficiency traits. Specifically, selection against AOTD would not influence ADG but may result in the improvement of both FCR and RFI values, and a favorable slight decrease in BFT and increase in LP. Additionally, selection for DD with a faster feeding rate may result in the improvement of ADG but may negatively influence AOTD, which may eventually result in a negative impact on other production and feed efficiency traits. Therefore, careful evaluation of the genetic correlation among feeding pattern, production, and feed efficiency traits before including them in the breeding program should always be considered to avoid these potential problems.

\section{Conclusions}

In this research, most of the studied feeding pattern traits were found to be moderately to highly heritable, indicating their rapid improvement once included in the breeding program. The addition of the common spatial pen effect had a substantial impact on the phenotypic variability of most feeding pattern traits in DD pigs. Based on the LRT1 value, animal model 3 , with an additional random litter and spatial pen effect, was more appropriate than model 1 for all feeding pattern traits, with the exception of AOTD. However, based on the LRT2 value, model 2 was more appropriate than model 3 for all feeding pattern traits, with the exceptions of ADFI and AFIV. Among feeding pattern traits, ADFI had a consistent genetic and phenotypic correlation with most production and feed efficiency traits. However, selection against ADFI may result in a negative impact on the growth rate of DD pigs. At the genetic level, the most interesting correlation was found 
in AOTD, which indicates that selection against this trait would not influence ADG but would probably improve FCR, reduce RFI, and increase LP. However, the corresponding phenotypic correlations of AOTD with production and feed efficiency traits were weak, probably due to the low residual or environmental correlation for these traits, which implies that improvements to the farm environment and management may also positively influence these correlated traits. At the genetic level, the feeding pattern traits showed potential in improving feed efficiency and production traits. However, further studies are needed to evaluate their impact at the phenotypic level. Moreover, to obtain more precise results in future related studies, a larger number of experimental animals is recommended.

Author Contributions: Conceptualization, K.-S.S. and K.G.S.; methodology, K.G.S.; formal analysis, K.G.S., B.I.L., D.-H.L., Y.-G.C. and Y.-N.S.; data curation, S.-H.K. and K.-S.S.; writing-original draft preparation, K.G.S.; writing-review and editing, B.I.L., S.-H.K. and K.-S.S.; visualization, K.-S.S.; supervision, K.-S.S.; project administration, K.-S.S. All authors have read and agreed to the published version of the manuscript.

Funding: This research received no external funding.

Institutional Review Board Statement: Ethical review and approval were waived for this study, since no animal experimentation was conducted and only data from commercial farms were used in our analysis.

Informed Consent Statement: Not Applicable.

Data Availability Statement: Not Applicable.

Acknowledgments: This work was supported by the research promotion program of Sunchon National University.

Conflicts of Interest: The authors declare no conflict of interest.

\section{References}

1. de Haer, L.C.; de Vries, A. Effects of genotype and sex on the feed intake pattern of group housed growing pigs. Livest. Prod. Sci. 1993, 36, 223-232. [CrossRef]

2. McSweeney, J. Evaluation of an electronic feeder system used in group-housed pigs. In Proceedings of the Animal Genetics and Breeding Unit-Pig genetic workshop, Armidale, Australia, 8 November 2001; pp. 44-52.

3. Labroue, F.; Guéblez, R.; Sellier, P. Genetic parameters of feeding behaviour and performance traits in group-housed Large White and French Landrace growing pigs. Genet. Sel. Evol. 1997, 29, 451-468. [CrossRef]

4. Langdon, J.M., II. The Genetics of Pig Feeding Behavior. Master's Thesis, North Carolina State University, Raleigh, NC, USA, 2015.

5. Hall, A.D. Electronic Feeders in the Genetic Improvement of Pigs for the Efficiency of Lean Growth; University of Edinburgh: Edinburgh, UK, 1997; Volume 1997.

6. Do, D.N.; Strathe, A.B.; Jensen, J.; Mark, T.; Kadarmideen, H.N. Genetic parameters for different measures of feed efficiency and related traits in boars of three pig breeds1. J. Anim. Sci. 2013, 91, 4069-4079. [CrossRef] [PubMed]

7. Lu, D.; Jiao, S.; Tiezzi, F.; Knauer, M.; Huang, Y.; Gray, K.A.; Maltecca, C. The relationship between different measures of feed efficiency and feeding behavior traits in Duroc pigs1. J. Anim. Sci. 2017, 95, 3370-3380. [CrossRef] [PubMed]

8. Kavlak, A.T.; Uimari, P. Estimation of heritability of feeding behaviour traits and their correlation with production traits in Finnish Yorkshire pigs. J. Anim. Breed. Genet. 2019, 136, 484-494. [CrossRef] [PubMed]

9. Noblet, J.; Karege, C.; Dubois, S.; van Milgen, J. Metabolic utilization of energy and maintenance requirements in growing pigs: Effects of sex and genotype. J. Anim. Sci. 1999, 77, 1208. [CrossRef]

10. Eissen, J.J.; Kanis, E.; Merks, J.W.M. Algorithms for identifying errors in individual feed intake data of growing pigs in grouphousing. Appl. Eng. Agric. 1998, 14, 667-673. [CrossRef]

11. Casey, D.S.; Stern, H.S.; Dekkers, J.C.M. Identification of errors and factors associated with errors in data from electronic swine feeders1. J. Anim. Sci. 2005, 83, 969-982. [CrossRef] [PubMed]

12. SAS Institute. SAS Version 9.4; SAS Institute Inc.: Cary, NC, USA, 2013.

13. Gilbert, H.; Bidanel, J.-P.; Gruand, J.; Caritez, J.-C.; Billon, Y.; Guillouet, P.; Lagant, H.; Noblet, J.; Sellier, P. Genetic parameters for residual feed intake in growing pigs, with emphasis on genetic relationships with carcass and meat quality traits. J. Anim. Sci. 2007, 85, 3182-3188. [CrossRef]

14. Saintilan, R.; Mérour, I.; Brossard, L.; Tribout, T.; Dourmad, J.Y.; Sellier, P.; Bidanel, J.; van Milgen, J.; Gilbert, H. Genetics of residual feed intake in growing pigs: Relationships with production traits, and nitrogen and phosphorus excretion traits1. J. Anim. Sci. 2013, 91, 2542-2554. [CrossRef] [PubMed] 
15. Meyer, K. WOMBAT-A tool for mixed model analyses in quantitative genetics by restricted maximum likelihood (REML). J. Zhejiang Univ. Sci. B 2007, 8, 815-821. [CrossRef]

16. Wolfinger, R. Covariance structure selection in general mixed models. Commun. Stat.-Simul. Comput. 1993, 22, 1079-1106. [CrossRef]

17. Von Felde, A.; Roehe, R.; Looft, H.; Kalm, E. Genetic association between feed intake and feed intake behaviour at different stages of growth of group-housed boars. Livest. Prod. Sci. 1996, 47, 11-22. [CrossRef]

18. Labroue, F.; Guéblez, R.; Sellier, P.; Meunier-Salaün, M. Feeding behaviour of group-housed large white and landrace pigs in french central test stations. Livest. Prod. Sci. 1994, 40, 303-312. [CrossRef]

19. Hoque, M.A.; Suzuki, K. Genetic parameters for production traits and measures of residual feed intake in Duroc and Landrace pigs. Anim. Sci. J. 2008, 79, 543-549. [CrossRef]

20. Hoque, M.A.; Kadowaki, H.; Shibata, T.; Oikawa, T.; Suzuki, K. Genetic parameters for measures of residual feed intake and growth traits in seven generations of Duroc pigs. Livest. Sci. 2009, 121, 45-49. [CrossRef]

21. Cheverud, J.M. Quantitative genetics and developmental constraints on evolution by selection. J. Theor. Biol. 1984, 110, 155-171. [CrossRef]

22. Rauw, W.M.; Soler, J.; Tibau, J.; Reixach, J.; Raya, L.G. The relationship between residual feed intake and feed intake behavior in group-housed Duroc barrows1. J. Anim. Sci. 2006, 84, 956-962. [CrossRef] [PubMed] 